\title{
Doping dependence of the Raman peaks intensity of graphene near the Dirac point
}

\author{
C. Casiraghi \\ Physics Department, Free University, Arnimallee 14, 14195 Berlin, Germany
}

(Dated: October 22, 2018)

\begin{abstract}
Here we use pristine graphene samples in order to analyze how the Raman peaks intensity, measured at $2.4 \mathrm{eV}$ and $1.96 \mathrm{eV}$ excitation energy, changes with the amount of doping. The use of pristine graphene allows investigating the intensity dependence close to the Dirac point. We show that the $\mathrm{G}$ peak intensity is independent on the doping, while the $2 \mathrm{D}$ peak intensity strongly decreases for increasing doping. Analyzing this dependence in the framework of a fully resonant process, we found that the total electron-phonon scattering rate is $\sim 40 \mathrm{meV}$ at $2.4 \mathrm{eV}$.
\end{abstract}

Graphene continues to attract interest because of its unique electronic properties [1, 2, 3], which make it a potential material for future nanoelectronics [4, 5]. Graphene layers can be readily identified by elastic and inelastic light scattering, such as Raman [6] and Rayleigh [7] spectroscopies. Raman spectroscopy is able to identify graphene and also to provide several information such as the amount of disorder [8], doping 9, 10, 11] and the atomic arrangements at the edges [12, 13]. Beside these practical applications, Raman spectroscopy in graphitic systems is extremely interesting because it involves resonant conditions [14, 15], defect-induced processes [14] and Kohn Anomalies (KA), which strongly affects the $\Gamma-E_{2 g}$ and $K-A_{1}^{\prime}$ modes [16].

All carbons show common features in their Raman spectra in the $800-2000 \mathrm{~cm}^{-1}$ region, the so-called $\mathrm{G}$ and D peaks, which lie at around 1580 and $1360 \mathrm{~cm}^{-1}$ respectively [17]. The D peak is due to the breathing modes of $\mathrm{sp}^{2}$ rings and requires a defect for its activation [18]. The $\mathrm{G}$ peak corresponds to the $E_{2 g}$ phonon at the Brillouin zone center [17]. The Raman spectra of graphite and graphene also show second-order scattering [6]. The $\mathrm{D}$ peak and its second order peak (2D) are activated by resonance processes 14, 15].

Raman Spectroscopy can easily monitor doping in graphene, as reported in refs. [10, 11, 19]. Here, the electron or hole concentration was directly controlled by applying a gate voltage, in top or back-gate configuration, which produces a shift of the Fermi energy $\left(E_{F}\right)$ from the Dirac point. The Raman spectrum shows the following variations with doping:

i) the $G$ peak position increases for increasing $\left|E_{F}\right|$ and saturates for high doping [10, 11, 19]. This is due to the nonadiabatic removal of the Kohn anomaly at $\Gamma[20]$;

ii) the G peak Full Width at Half Maximum decreases for increasing $\left|E_{F}\right|$, and saturates when the electron-hole gap becomes higher than the phonon energy [11, 20]. This is due to the blockage of the phonon decay into electron-hole pairs due to the Pauli exclusion principle [20].

iii) the $2 \mathrm{D}$ peak position increases for p-doping, while it decreases for n-doping, for increasing $\left|E_{F}\right|[10]$. The 2D and $\mathrm{G}$ peaks show a different doping dependence because the nonadiabatic effects are expected to be negligible on the $2 \mathrm{D}$ phonons, when measuring with visible energy [10].

iv) the ratio between the peaks intensity, $\mathrm{I}(2 \mathrm{D}) / \mathrm{I}(\mathrm{G})$, decreases for increasing $\left|E_{F}\right|[10]$. In the framework of the fully Raman resonant process for the second order $2 \mathrm{D}$ peak, the intensity dependence on the doping is due to the electron-electron scattering contribution, which increases for increasing charge concentration [22].

The same variations have been observed in the Raman spectrum of several pristine graphene samples, deposited on $\mathrm{Si} / \mathrm{SiO}_{x}$ substrate and they have been attributed to doping by charged impurities [9]. Pristine graphene samples can have charge concentration up to $10^{-13} \mathrm{~cm}^{-2}$, and they are usually p-doped [8, 9]. Unintentional doping in graphene deposited on $\mathrm{Si} / \mathrm{SiO}_{x}$ was first observed in gating experiments: the $\mathrm{G}$ peak position obtained for $E_{F}=0$ did not correspond to the $G$ peak position of an undoped graphene $\left(\sim 1580 \mathrm{~cm}^{-1}\right.$, see Fig. 4 in ref. [19]). Thus, it is not possible to reach the Dirac point by gating due to local charge density variations 19]. Recent works [23, 24, 25] have finally confirmed the presence of charged impurities in graphene. In particular, Raman and transport measurements performed on suspended graphene have shown that by eliminating the substrate it is possible to produce graphene samples, which are essentially undoped, have little disorder and the highest mobility [23, 26].

Here we analyze the absolute intensity of the $G$ and $2 \mathrm{D}$ peak, $\mathrm{I}(\mathrm{G})$ and $\mathrm{I}(2 \mathrm{D})$ respectively, of several pristine graphene samples with different amount of doping. The use of pristine graphene samples presents the following advantages: i) low doping level can be investigated, in contrast to gated-graphene; ii) graphene is not in a device-configuration, so contacts and lithography processes do not affect the properties of graphene [9]; iii) the sample is stable and it cannot be damaged by electrostatic charge or high voltage. This is particular important when measuring the absolute Raman intensity, since these measurements can be drastically altered as a result of surface conditions and strongly depend on the experimental set-up [27]. We will show that $\mathrm{I}(\mathrm{G})$ is insensitive to the doping, in contrast to I(2D), which strongly depends on the Fermi energy, as expected in the framework of the fully resonant activation process for the $2 \mathrm{D}$ peak [15].

We study several graphene samples, produced by micro-mechanical cleavage of bulk graphite and deposited on $\mathrm{Si} / \mathrm{SiO}_{x}$. Only single layers flakes that completely fills the laser focus $\left(\sim 1 \mu \mathrm{m}^{2}\right)$ were selected in order to 


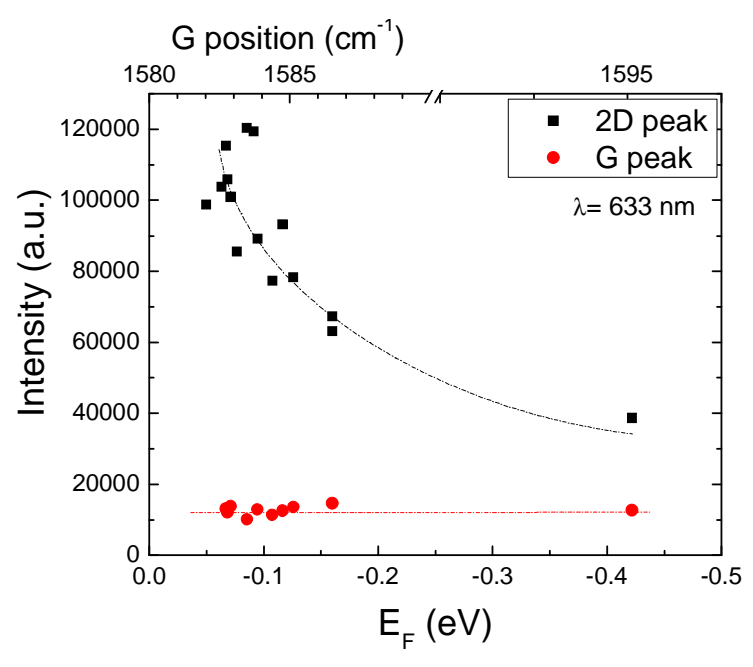

FIG. 1: (Color online) G and 2D peak intensity as a function of the Fermi energy and $G$ peak position, measured at 633 $\mathrm{nm}$. The dotted lines are a guide for the eyes.

avoid the graphene edges, since they can strongly affect the intensity [12]. Unpolarized Raman spectra are measured at $633 \mathrm{~nm}$ and $514 \mathrm{~nm}$ by using a Renishaw microRaman 1000 spectrometer. The Raman spectra are collected with a 100X objective and the spectral resolution is $\sim 3 \mathrm{~cm}^{-1}$. The power on the samples is always kept well below $2 \mathrm{~mW}$. The spectra have been fitted by using a Lorentzian spectral shape for both $\mathrm{G}$ and $2 \mathrm{D}$ peaks and the intensity is calculated as integrated area. The Raman spectra of the samples do not show any D peak, indicating a high crystallinity. In the case of pristine graphene samples, the amount of doping is not directly accessible through a gate voltage. Thus, we used the dependence of the $\mathrm{G}$ peak position on the doping in order to derive the Fermi energy for every sample. We used the relations between $\mathrm{G}$ peak positions and $E_{F}$ calculated for an ideal graphene at $300 \mathrm{~K}$ in ref. [20].

Figure 1 correlates $\mathrm{I}(\mathrm{G})$ and $\mathrm{I}(2 \mathrm{D})$ with $E_{F}$. This figure clearly shows that $\mathrm{I}(\mathrm{G})$ is insensitive to changes in $E_{F}$, in agreement with the predictions in ref. 21]. In contrast, I(2D) strongly decreases for increasing doping. This is in agreement with the Raman theory, under the assumption of a fully resonant process and for $E_{F}<<1$ $\mathrm{eV}$ [22]. Assuming two main scattering mechanisms, i.e. the emission of phonons $\left(\gamma_{e p}\right)$ and the electron-electron collision $\left(\gamma_{e e}\right), \mathrm{I}(2 \mathrm{D})$ can be written as [22]:

$$
I(2 D)=C\left(\gamma_{K} / \gamma\right)^{2}
$$

where $\mathrm{C}$ is a constant and $2 \gamma$ is the total scattering rate, so $\gamma=\gamma_{e p}+\gamma_{e e}$ and $\gamma_{e p}=\gamma_{K}+\gamma_{\Gamma}$. Note that the scattering rate $\gamma_{K}$ and $\gamma_{\Gamma}$ depends on the incident energy [22].

It has been shown that only $\gamma_{e e}$ depends on $E_{F}$, so eq. 1 can be written as:
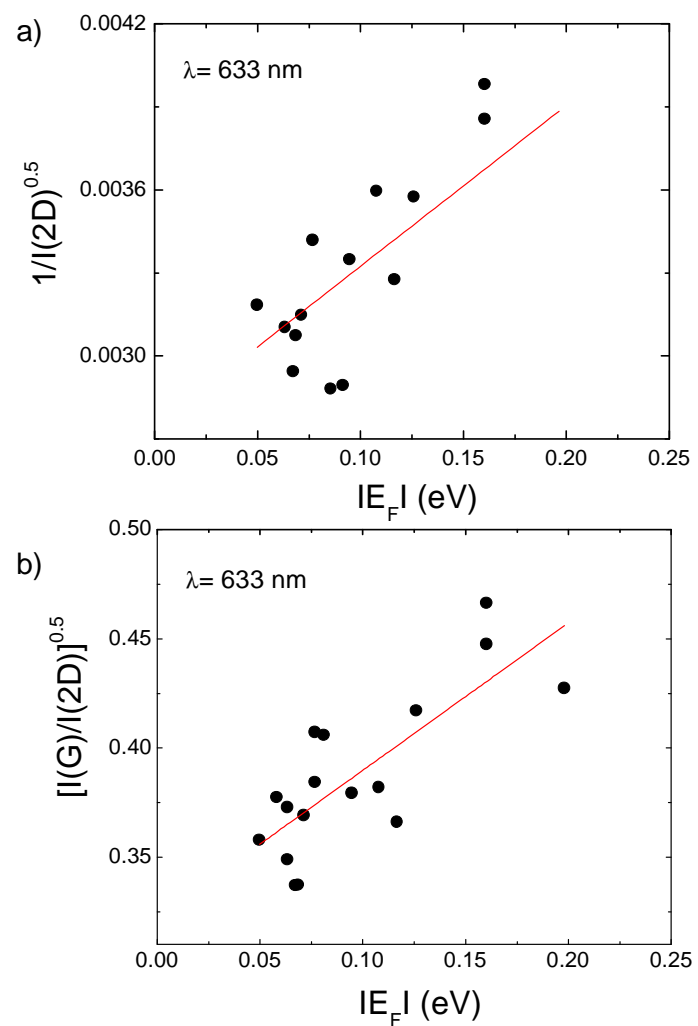

FIG. 2: (Color online) Fit of the experimental dependence: (a) $1 / \sqrt{I(2 D)}$ and (b) $1 / \sqrt{I(G) / I(2 D)}$ as a function of the Fermi energy, measured at $633 \mathrm{~nm}$. Both fits give $\gamma_{e p} \sim 30$ $\mathrm{meV}$.

$$
\sqrt{1 / I(2 D)}=\frac{1}{\gamma_{K} \sqrt{C}}\left(\gamma_{e p}+f(\varepsilon)\left|E_{F}\right|\right)
$$

where $\mathrm{f}$ is a function which depends on the dielectric environment 22]. In our case, the dielectric constant $\varepsilon$ for $\mathrm{SiO}_{x}$ is 4.5 , so $f \simeq 0.07$ [22]. Thus, eq. 2 shows that $\mathrm{I}(2 \mathrm{D})$ decreases for increasing $E_{F}$, as observed in Figure 1.

Eq. 2 can be used to derive the electron-phonon scattering rate by measuring the variation of the absolute intensity of the 2D peak with doping. However, this requires to compare the absolute Raman intensity, i.e. to measure all the spectra under exactly the same experimental conditions. This is not always possible, in particular, during gating experiments. Since $I(G)$ is insensitive to the doping (Fig. 1), $\mathrm{I}(\mathrm{G}) / \mathrm{I}(2 \mathrm{D})$ can be used in order to derive $\gamma_{e p}$ since:

$$
\sqrt{I(G) / I(2 D)}=C^{\prime}\left(\gamma_{e p}+0.07\left|E_{F}\right|\right)
$$

where $\mathrm{C}^{\prime}$ is another constant. Eq. 3 has been used to find $\gamma_{e p}$ of gated-graphene [22]. There, values ranging from 18 to $65 \mathrm{meV}$, with an average of $\sim 33 \mathrm{meV}$, 


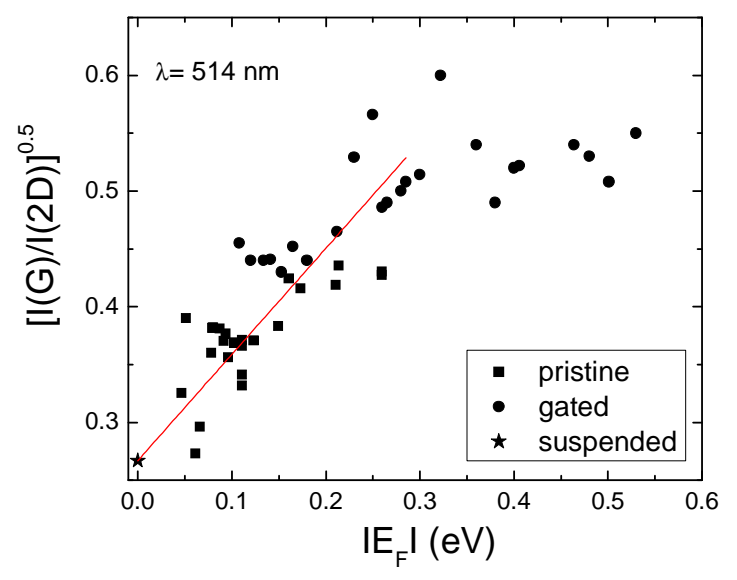

FIG. 3: (Color online) Fit of the experimental dependence $1 / \sqrt{I(G) / I(2 D)}$ as a function of the Fermi energy for pristine graphene, measured at $514 \mathrm{~nm}$. The data for suspended graphene 23] and the back-gated graphene 22] have been included in the figure.

have been reported, depending on the data set 22]. In particular, for a hole-doped graphene a good consistency between different data has been found, resulting in $\gamma_{e p} \sim$ $20 \mathrm{meV}$ at $514 \mathrm{~nm}$ [22]. Here we use eqs 2 and 3 in order to find $\gamma_{e p}$ using pristine graphene samples. The present data, even though collected from many samples, are much less scattered than the data obtained by gating experiments, in particular close to the Dirac point, where eqs. 2 and 3 are well valid. Thus, our data enable determining $\gamma_{e p}$ with a significantly smaller uncertainty.
Figure 2 (a) shows the linear fit of the experimental dependence $\sqrt{1 / I(2 D)}$ on the doping (eq. 2), measured at $633 \mathrm{~nm}$. We found: $\gamma_{e p}=33 \mathrm{meV}$. The linear fit of the experimental dependence $\sqrt{I(G) / I(2 D)}$ on the doping (eq. 3), measured at $633 \mathrm{~nm}$, is shown in Figure 2(b) and gives again $\gamma_{e p}=33 \mathrm{meV}$. Thus, for an intrinsic graphene, i.e. for $E_{F}=0$, the fit in Fig. 2 (b) gives $\mathrm{I}(2 \mathrm{D}) / \mathrm{I}(\mathrm{G}) \sim$ 10.

Figure 3 shows the linear fit of the experimental dependence $\sqrt{I(G) / I(2 D)}$ on the doping (eq. 3), measured at $514 \mathrm{~nm}$. Here, we included the data obtained for suspended graphene, by constraining the fit and we obtained $\gamma_{e p}=21 \mathrm{meV}$. Furthermore, we included in Figure 3 the data obtained for graphene in back-gate configuration for $\left|E_{F}\right|>0.1 \mathrm{eV}$, as taken from ref. 22]. Figure 3 clearly shows that eq. 3 is valid up to $E_{F}=0.3-0.4 \mathrm{eV}$, as expected, since eqs. 2 and 3 are valid only for $E_{F}<<1 \mathrm{eV}$.

In conclusion, we have shown that the $G$ peak intensity is independent on the doping, while the 2D peak intensity is strongly sensitive to the dynamics of the photoexcited electron-hole pairs. By measuring the dependence of $\mathrm{I}(2 \mathrm{D})$ on the Fermi energy close to the Dirac point, we have found that the total electron-phonon scattering rate $\left(2 \gamma_{e p}\right)$ of graphene is $\sim 40 \mathrm{meV}$ at $2.4 \mathrm{eV}$, in good agreement with the hole-doping side of the top and back-gated graphene experiments 22].

The author acknowledges D. M. Basko for inspiring discussions and critical reading of the manuscript. This work was supported by the Alexander von Humboldt Foundation in the framework of the Sofja Kovalevskaja Award, endowed by the Federal Ministry of Education and Research.
[1] K. S. Novoselov, A. K. Geim, S. V. Morozov, D. Jiang, M. I. Katsnelson, I. V. Grigorieva, S. V. Dubonos, and A. A. Firsov; Nature (London), 438, 197 (2005).

[2] Y. Zhang, Y.W. Tan, H. L. Stormer, and P. Kim; Nature (London), 438, 201 (2005).

[3] S. V. Morozov, K. S. Novoselov, M. I. Katsnelson, F. Schedin, D. C. Elias, J. A. Jaszczak, A. K. Geim; Phys. Rev. Lett., 100, 016602 (2008).

[4] Z. Chen, Y. M. Lin, M. Rooks, P. Avouris, Physica E, 40, 228 (2007)

[5] M. C. Lemme, T. J. Echtermeyer, M. Baus, H. Kurz, IEE El. Dev. Lett, 28, 4 (2007)

[6] A. C. Ferrari, J. C. Meyer, V. Scardaci, C. Casiraghi, M. Lazzeri, F. Mauri, S. Piscanec, D. Jiang, K. S. Novoselov, S. Roth, A. K. Geim, Phys. Rev. Lett., 97, 187401 (2006)

[7] C. Casiraghi, A. Hartschuh, E. Lidorikis, H. Qian, H. Harutyunyan, T. Gokus, K. S. Novoselov, A. C. Ferrari; Nano. Lett., 7, 2711 (2007).

[8] C. Casiraghi, Phys. Status Solidi RRL, DOI 10.1002/pssr.200903135

[9] C. Casiraghi, S. Pisana, K. S. Novoselov, A. K. Geim, A. C.Ferrari; Appl. Phys. Lett., 91, 233108 (2007).

[10] A. Das, S. Pisana, S. Piscanec, B. Chakraborty, S. K.
Saha, U. V. Waghmare, R. Yang, H. R. Krishnamurhthy, A. K. Geim, A. C. Ferrari, A. K. Sood Nature Nanotech. 3, $210(2008)$

[11] S. Pisana, M.Lazzeri, C. Casiraghi, K. Novoselov, A. K. Geim, A. C. Ferrari, F. Mauri, Nature Mat. 6, 198 (2007)

[12] C. Casiraghi, A. Hartschuh, H. Qian, S. Piscanec, C. Georgi, A. Fasoli, K. S. Novoselov, D. M. Basko, A. C. Ferrari, Nano Lett. 9, 1433 (2009)

[13] D. M. Basko, Phys. Rev. B, 79, 205428 (2009)

[14] C. Thomsen, S. Reich, Phys. Rev. Lett. 85, 5214 (2000)

[15] D. M. Basko, Phys. Rev. B, 78, 125418 (2008)

[16] S. Piscanec, M. Lazzeri, F. Mauri, A. C. Ferrari, J. Robertson, Phys. Rev. Lett. 93, 185503 (2004)

[17] A. C. Ferrari, J. Robertson (eds), Raman spectroscopy in carbons: from nanotubes to diamond, Theme Issue, Phil. Trans. Roy. Soc. A 362, 2267-2565 (2004)

[18] F. Tuinstra, J. L. Koenig, J. Chem. Phys. 53, 1126 (1970)

[19] J. Yan, Y. Zhang, P. Kim, A. Pinczuk, Phys. Rev. Lett. 98, $166802(2007)$

[20] M. Lazzeri, F. Mauri, Phys. Rev. Lett. 97, 266407 (2006)

[21] D. M. Basko, New J. Phys., in press

[22] D. M. Basko, S. Piscanec, A. C. Ferrari, arXiv: 0906.0975

[23] S. Berciaud, S. Ryu, L. E. Brus, T. F. Heinz, Nano Lett. 
9, $346(2009)$

[24] Z. H. Ni, T. Yu, Z. Q. Ting, Z. Q. Luo, Y. Y. Wang, L. Liu, C. P. Wong, J. M. Miao, W. Huang, Z. X. Shen, ACS Nano, 3, 569 (2009)

[25] Y. Shi, X. Dong, P. Chen, J. Wang, L-J. Li, Phys. Rev. B, 79, 115402 (2009)
[26] Bolotin, K. I., Sikes, K. J., Jiang Z., Klima M., Fudenberg G., Hone J., Kim P., Stormer H. L., Solid State Commun. 146, 351 (2008)

[27] C. Casiraghi, Ph. Klar, E. Lidorikis, R. Panknin, S. Reich, submitted 